\title{
Das deutsche Bronchiektasenregister PROGNOSIS rekrutiert die ersten Patienten
}

Seit Ende Juni dieses Jahres werden die ersten Patienten in das deutsche Bronchiektasenregister PROGNOSIS eingeschlossen. „Nach einem Vorlauf von ca. 3 Jahren sind wir sehr glücklich, endlich die so wertvollen Daten unserer Bronchiektasenpatienten systematisch in einem spezifischen Register erfassen zu können“, sagt Dr. Felix C. Ringshausen, Oberarzt in der Klinik für Pneumologie der Medizinischen Hochschule Hannover (MHH) und einer der Initiatoren von PROGNOSIS.

Der Weg war lang und steinig. Bronchiektasen sind mit Schleim und Eiter gefüllte Aussackungen der Atemwege, die zu starkem Husten, Ausgezehrtheit und chronischer Entzündung führen. Trotz des hohen Leidensdrucks der Patienten und der häufig aufwändigen und zeitintensiven Therapie fand diese Erkrankung lange Zeit wenig Aufmerksamkeit. Zwar werden Bronchiektasen in Verbindung mit einer Mukoviszidose (engl. Cystic Fibrosis, CF) schon seit langer Zeit gezielt behandelt, wenn sie aber nicht mit einer $\mathrm{CF}$ in Verbindung stehen, es sich also um sog. Non-CF-Bronchiektasen handelt, fielen sie lange Zeit in die Kategorie der seltenen Erkrankungen (definiert als Prävalenz von < 5 auf 10000 Personen).

Dass die Prävalenz jedoch deutlich höher ist, konnte jetzt gezeigt werden: Dr. Ringshausen und eine Gruppe von Ärzten, die

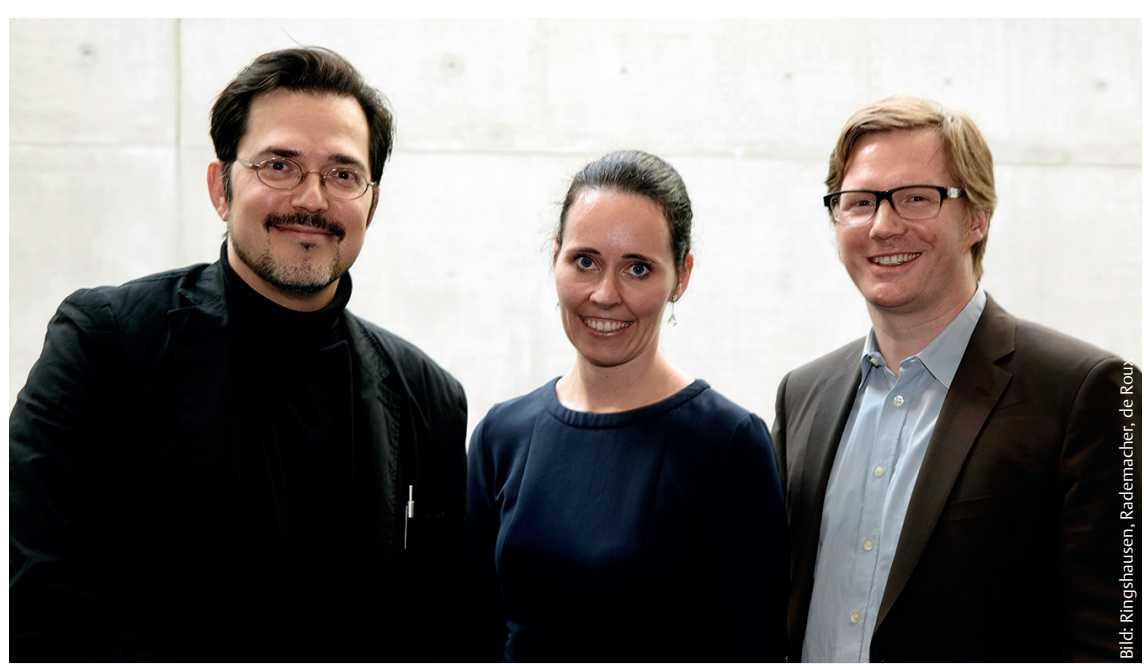

Initiatoren und Vorstand von PROGNOSIS (v. r. n. I.): Dr. Felix C. Ringshausen, Dr. Jessica Rademacher,

beide Hannover, und Dr. Andrés de Roux, Berlin.

sich des Themas Non-CF-Bronchiektasen angenommen haben und seit Jahren unermüdlich am Aufbau eines Registers arbeiten, veröffentlichten kürzlich eine Analyse von Krankenkassendaten, die zeigt, dass die Prävalenz in Deutschland im Jahr 2013 bei 6,7 auf 10000 Personen lag. Somit können Bronchiektasen keineswegs zu den seltenen Erkrankungen gezählt werden. Die überwiegende Mehrheit der Patienten wurde ambulant behandelt, und mehr als die Hälfte der Erkrankten litt außerdem an einer chronisch obstruktiven Ventilationsstörung. Da das Bewusstsein für diese Krankheit weltweit stark auf dem Vormarsch ist, muss damit gerechnet werden, dass Bronchiektasen in Zukunft sogar noch häufiger diagnostiziert werden.

Vor 3 Jahren trafen sich das Ärzteteam um Dr. F. Ringshausen zum ersten Mal, um das Thema Non-CF-Bronchiektasen systematisch anzugehen und durch den Aufbau eines Registers die Situation ihrer Patienten mittelfristig zu verbessern. 17 Gründungszentren zählt PROGNOSIS heute, unter ihnen auch etliche niedergelassene Lungenfachärzte. Gerade im ambulanten Bereich stellt die Betreuung von Non-CFBronchiektasenpatienten eine besondere Herausforderung dar. „Therapeutische Konzepte müssen häufig individuell auf den Patienten zugeschnitten werden. Dabei müssen wir in Ermangelung von Alter- nativen häufig Therapien einsetzen, für die es keine formale Zulassung gibt oder die sehr hochpreisig sind“, sagt Dr. Andres de Roux, niedergelassener Pneumologe aus Berlin und Mitglied im Vorstand von PROGNOSIS.

Dass sich die Rekrutierung der ersten Patienten für das Register so lange hingezogen hat, lag wie so oft an der fehlenden Finanzierung. „Aber wir haben trotzdem weitergemacht und alle Voraussetzungen für das Register auch ohne Funding so weit wie irgendmöglich vorangetrieben“, sagt Dr. Jessica Rademacher, von der $\mathrm{MHH}$ und zentral an der Initiierung von PROGNOSIS beteiligt. „Hierbei haben uns v.a. die CAPNETZ STIFTUNG und das Forschungsnetzwerk BREATH, Standort Hannover des Deutschen Zentrums für Lungenforschung (DZL), unterstützt.“

Im europäischen Kontext ist man hier schon etwas weiter: Das europäische Bronchiektasenregister EMBARC konnte mit Hilfe einer Finanzierung im Sinne einer Public Private Partnership durch EUGelder und Beteiligung der Industrie bereits Anfang des Jahres mit der Rekrutierung von Patienten starten. PROGNOSIS wird seine Patientendaten in Zukunft nun ebenfalls in EMBARC einbringen, um diese auch im europäischen Kontext erfassen und analysieren zu können.

Ende Juli kam dann auch die erlösende Nachricht: der Vertrag mit Bayer Vital zur entscheidenden Unterstützung von PROGNOSIS für die nächsten 3 Jahre wurde nach intensiver Verhandlungsphase unterzeichnet. „Endlich können wir durchstarten“, sagt Dr. Ringshausen. „Es wird wirklich Zeit, dass wir valide Daten zu dieser Erkrankung sammeln. Auf Basis der Ergebnisse möchten wir u.a. deutschsprachige Leitlinien für die Diagnostik sowie Prognosemarker für Non-CF-Bronchiektasen entwickeln, um die Versorgung unserer Patienten nachhaltig zu verbessern“.

Die Zahl der Zentren, die Patienten in das PROGNOSIS-Register einschließen, soll in den nächsten 3 Jahren auf mindestens 25 ansteigen. Interessierte Ärzte können sich im Internet über www.bronchiektasenregister.de weiter informieren und wenden sich für einen persönlichen Kontakt bitte per E-Mail an prognosis@capnetz.de.

Nach einer Mitteilung von BREATH/DZL, Hannover 